\title{
BOUNDED MEAN OSCILLATION AND REGULATED MARTINGALES(1)
}

\author{
BY
}

\section{CARL HERZ}

\begin{abstract}
In the martingale context, the dual Banach space to $H_{1}$ is BMO in analogy with the result of Charles Fefferman [4] for the classical case. This theorem is an easy consequence of decomposition theorems for $H_{1}$-martingales which involve the notion of $L_{p}$-regulated $L_{1}$-martingales where $1<p \leq \infty$. The strongest decomposition theorem is for $p=\infty$, and this provides full information about BMO. The weaker $p=2$ decomposition is fundamental in the theory of martingale transforms.
\end{abstract}

Introduction. Shortly after Charles Fefferman [4] proved that the dual Banach space to the Hardy space $H_{1}$ (in this context we view $H_{1}$ as the space of functions on the circle which together with their conjugate function belong to $L_{1}$ ) was equivalent to the space BMO of functions of bounded mean oscillation treated by John and Nirenberg [9], a martingale analogue was proved by Fefferman and Stein, A. Garsia, and me; see [5] and [6]. In this Richard Gundy played a role which, at least for me, was of the highest value. There are related matters in [7] and [11].

The martingale spaces $\mathrm{BMO}_{p}$ are defined for $1 \leq p<\infty$ by the John-Nirenberg conditions $\mathrm{JN}_{p}$ plus a supplementary condition which is needed to handle the case of general martingales; see $\$ 2$ below for the definitions. In the classical case, that of martingales on a dyadic stochastic base, John and Nirenberg proved that, while the defining conditions for membership in $\mathrm{BMO}_{p}$ appear to be more stringent with increasing $p$, they are all equivalent. The John-Nirenberg results remain valid for general martingales; the statement is Theorem JN in $\$ 2$. Although this theorem is stated right after a section of preliminaries and the basic definitions, it is one of the deepest results in the paper and much of the subsequently developed machinery is used in the proof.

The duality of $H_{1}$ with BMO in both the classical and martingale cases is proved in two parts. The easy step is to show that every bounded linear functional

Received by the editors August 30, 1973.

AMS (MOS) subject classifications (1970). Primary 30A78, 60G45; Secondary 44A25.

Key words and phrases. Martingales, bounded mean oscillation, Hardy class, maximal function, martingale contraction, conjugate function.

(1) The research for this article was supported by the National Research Council of Canada, Grant No. A7678.

Copyrieht $\odot$ 1974, American Mathematical Soctoty 
on $H_{1}$ is represented by a "function" in $\mathrm{BMO}_{p}$ for every $p$. This result we call Theorem E to suggest "exponential class". The idea is quite simple. For probability spaces one has that the $H_{1}$-norm is dominated by the $L \log L$ norm; the bounded linear functionals on $H_{1}$ must also be bounded on $L \log L$ and hence belong to the exponential class. The conditional version of this statement is Theorem E; see $\$ 3$ for the precise details.

The hard part of the duality theorem is to prove Theorem $F_{p}$ : the elements of $\mathrm{BMO}_{p}$ give bounded linear functionals on $H_{1}$. The Fefferman result in the classical case and the first martingale generalization would correspond to Theorem $\mathrm{F}_{2}$. Here we prove the stronger Theorem $F_{1}$. In the classical case $F_{1}$ is a corollary of $F_{2}$ via the John-Nirenberg Theorem JN. In our approach, Theorem JN for general martingales is a corollary of $F_{1}$ via Theorem $\mathrm{E}$.

In this article $I$ have tried to cast my proofs of Theorems $F_{1}$ and $F_{2}$ in a form which I hope will illuminate the subject. (Theorem $F_{2}$ is an immediate corollary of $F_{1}$, but it has a simple independent proof which is of interest in its own right.) The basic idea is to define certain spaces ${ }_{p} L_{1}$ of " $L_{p}$-regulated $L_{1}$-martingales". These have a vague analogy with some spaces of Beurling discussed in [8]. The ${ }_{p} L_{1}$ spaces seem of natural interest in martingale theory, and, what is crucial here, it is an abstract triviality that a "function" satisfying the JohnNirenberg conditions $\mathrm{JN}_{p^{\prime}}$ gives a bounded linear functional on ${ }_{p} L_{1}$ ( $p^{\prime}$ is always the conjugate index to $p$, that is $1 / p+1 / p^{\prime}=1$ ).

The ${ }_{p} L_{1}$-spaces are defined in $\$ 5$, but we shall give a rough indication here. Let $\psi$ be an $L_{p}$-function and $\left\{\mathcal{E}_{n} \psi\right\}$ the martingale sequence formed by its successive conditional expectations. Suppose that for some integer $N$ we have $\varepsilon_{N} \psi=0$ while $g$ is an $L_{p^{\prime}}$-function which depends on the past up to time $N$. Then the product $g \psi$ is a prototypical element of ${ }_{p} L_{1}$. Note that for $f=g \psi$ we have $\tilde{E}_{n} f=$ $g \tilde{E}_{n} \psi$, so that the successive fortunes $\left\{\tilde{E}_{n} f\right\}$ are the fortunes in a fair $L_{p}$-game starting at time $N+1$, namely the sequence $\left\{\tilde{E}_{n} \psi\right\}$, multiplied by an $L_{p^{\prime}}$ initial bet, $g$. The Banach space ${ }_{p} L_{1}$ consists of appropriate norm-convergent sums of these prototypical elements. The spaces ${ }_{p} L_{1}$ for $1<p \leq \infty$ form a family of subspaces of $H_{1}$ which decrease as $p$ increases.

Theorem $\mathrm{F}_{1}$ is a rather easy consequence of Theorem $\mathrm{B}$, a decomposition theorem for $H_{1}$-martingales given in $\$ 8$. The definition of the $H_{1}$-class used here is in terms of the martingale maximal function. We say that $b \in H_{1}$ if $M b \in L_{1}$ where $M b=\sup _{n}\left|\mathcal{E}_{n} b\right|$; there is also a normalizing assumption, $\lim _{n \rightarrow-\infty} \mathcal{E}_{n} b=0$. What Theorem $\mathrm{B}$ asserts is that any $b \in H_{1}$ can be split as $b=a+f$ where $\Sigma\left\|\varepsilon_{n} a-E_{n-1} a\right\|_{1}<\infty$ and $f \epsilon_{\infty} L_{1}$.

The proof of Theorem B begins with an idea from Burgess Davis [3]. One way to formulate Davis' device is to say that an $H_{1}$-martingale can be split as $b=a+f$ where $a$, the part having the big jumps in the conditional expectations, is as before, 
and $f$ corresponds to a martingale having majorisations $\left|E_{n} f\right| \leq r_{n-1}$ where $\left\{r_{n}\right\}$ is an increasing $L_{1}$-bounded sequence such that $r_{n}$ depends only on the past up to time $n$. Thus the condition $\left|\mathcal{E}_{n} f\right| \leq r_{n-1}$ means we have a good control on $\mathscr{E}_{n} f$ in terms of the strict past.

The key result is Theorem $A_{\infty}$ in $\$ 6$ which states that the martingales having the above property are precisely the $L_{\infty}$-regulated $L_{1}$-martingales previously defined by saying $f \epsilon_{\infty} L_{1}$. This is what is needed to conclude the proof of Theorem B, and it gives a characterization of ${ }_{\infty} L_{1}$ in useful terms. The proof of Theorem $A_{\infty}$ is the only place one has to work hard and the only place where we use genuinely probabilistic techniques.

Theorem $A_{2}$ in $\$ 7$ characterizes the space ${ }_{2} L_{1}$ of $L_{2}$-regulated $L_{1}$-martingales in familiar terms. The point is that the ${ }_{p} L_{1}$-spaces are defined in a way which is technically convenient, but this definition gives no insight how to verify in practice that a function belongs to the space.

Theorem $\mathrm{B}_{2}$ is the weakened version of Theorem $\mathrm{B}$ which states that $b=a+f$ where now $f \epsilon_{2} L_{1}$. The space ${ }_{2} L_{1}$ is preserved by a large class of martingale transformations. As a consequence of Theorem $B_{2}$ we have Theorem $C$ in $\$ 4$ which states that $H_{1}$ is preserved under a wide class of martingale conjugate functions. By enlarging the notion of "conjugate function" to include Hilbert space-valued functions (this requires no extra effort) we can exhibit Davis' theorem as an immediate corollary, Theorem $\mathrm{D}$, of the preceding Theorem $\mathrm{C}$.

A remark about constants. When an estimate is given in the form $x \leq 6 y$ it means that the constant 6 may be the best possible. By contrast, $x<6 y$ means there is an estimate $x \leq c y$ with $c<6$. The result would be stated in this form had we in fact proved $c \leq 3+2 \sqrt{2}$. The effect is that in a subsequent calculation $x<12 y$ might be replaced by $9 x<53 y$. It also occurs that from a theorem $x_{p}<$ $53 y_{p}$ for all $p$, we state a corollary in the form $x_{2}<13 y_{2}$; what this indicates is that there is a way to prove $x_{2}<13 y_{2}$, but it is not worth explaining in view of the limited interest of the more refined estimate. On the other hand, there is an attempt to give good estimates, and if one of the displayed theorems asserts an inequality $x<6 y$ it means either the author is unable to prove $x \leq 5 y$ or that such a minor improvement would require a lot of extra work.

1. Preliminary notions. A measure ring $(\mathscr{Q}, \mu)$ is a pair consisting of a Boolean ring $\mathfrak{Q}$ and a function $\mu: \mathbb{Q} \rightarrow[0, \infty)$ such that $\mu(\varnothing)=0$ and $\mu(A \cup B)+\mu(A \cap B)=$ $\mu(A)+\mu(B)$ with the property that $\mathbb{Q}$ is a complete metric space when endowed with the distance $d(A, B)=\mu(A \Delta B)$ the symbol $\Delta$ standing for symmetric difference. We call the elements of $\mathbb{Q}$ " "events", but $\mu$ is not required to be a probability measure. The events all have finite measure and $\mu(A)>0$ unless $A=\varnothing$. This means we do not consider "sets" of infinite measure, and we throw away "sets" of measure 0 . This avoids having to write "almost everywhere" almost everywhere. 
A stochastic base for $(\mathfrak{Q}, \mu)$ is an increasing sequence $\left\{\mathfrak{Q}_{n}: n \in Z\right.$ \} of closed subrings of $\mathbb{Q}$ such that $\cup \mathbb{Q}_{n}$ is dense in $\mathbb{Q}$ and the following condition holds: for each $E \in \mathbb{Q}$, each $n \in \mathbb{Z}$, and each $\epsilon>0$, there exists $A \in \mathbb{Q}_{n}$ with $\mu(E \cap A)>$ $\mu(E)-\epsilon$.

We make no distinction between an event $A$ and its indicator function. A simple function is a linear combination of events and $L_{p}(\mathfrak{Q}, \mu ; \mathbf{R})$ is, for $1 \leq p<\infty$,

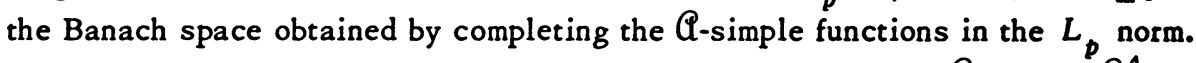
The space $L_{\infty}$ can be defined in the same spirit. For each $A \in \mathbb{Q}$ we put $\mathbb{Q}^{A}$ for the Boolean $\sigma$-algebra constituted by the subsets of $A$. The projective limit of the $\mathbb{Q}^{A}$ gives a Boolean $\sigma$-algebra $\widetilde{\mathfrak{Q}}$, and $L_{\infty}\left(\widetilde{\mathfrak{Q}}_{;} \mathbf{R}\right)$ consists of the bounded $\widetilde{\mathfrak{Q}}$-measurable functions. ( $\mathbb{Q}$ corresponds to the sets of finite measure in $\mathbb{Q}$.) For simplicity we assume that $(\mathscr{Q}, \mu)$ is $\sigma$-finite, i.e. the 1 -element of $\widetilde{Q}$ is a countable union of elements of $\mathbb{Q}$.

Excess baggage in the notation will be dropped where no unintended confusion can result.

The spaces $L_{p}\left(\mathscr{Q}_{m}\right)$ are included in $L_{p}(\mathfrak{Q})$. There is a unique retraction $\xi_{m}: L_{p}(\mathfrak{Q})-L_{p}\left(\mathfrak{Q}_{m}\right)$ with the properties

(i) $\int_{A} \mathcal{E}_{m} f d \mu=\int_{A} f d \mu$ for all $A \in \mathbb{Q}_{m}$ and $f \in L_{p}(\mathfrak{Q})$;

(ii) $\mathcal{E}_{m}(A f)=A \mathcal{E}_{m} f$ for all $A \in \mathbb{Q}_{m}$ and $f \in L_{p}(\mathfrak{Q})$.

(Remember that $A f$ is the function which equals $f$ on $A$ and 0 elsewhere.) The retractions $\mathcal{E}_{m}=\mathcal{G}\left\{: \mathbb{Q}_{m}\right\}$ are called "conditional expectations".

The symbol $L_{p}^{(m)}(\mathfrak{Q})$ denotes the subspace of $L_{p}(\mathfrak{Q})$ constituted by the elements $f \in L_{p}(\mathfrak{Q})$ with $\mathcal{E}_{m} f=0$.

A martingale (with respect to the sequence $\left\{\mathfrak{Q}_{n}, \mu\right\}$ ) is a sequence $\left\{f_{n}\right\}$ with each $f_{n}$ being an $\widetilde{\mathbb{Q}}_{n}$-measurable function integrable over all $A \in \mathbb{Q}$ with the property that $\mathscr{E}_{m} f_{n}=f_{m}$ whenever $m \leq n$. We shall only be concerned with martingales of the form $\left\{\tilde{G}_{n} f\right\}$ where $f \in L_{p}(\mathfrak{Q})$ for some $p$.

We always have $\left\|E_{n} f\right\|_{p} \leq\|f\|_{p}$ and $\int \mathcal{E}_{n} f d \mu=\int f d \mu$.

It is vital in what follows that we treat vector-valued functions; at least we need to allow values in a separable Hilbert space. For the most part no changes in arguments are needed to treat functions with values in an arbitrary Banach space $H$. For $1 \leq p<\infty$ we define $L_{p}(\mathbb{Q}, \mu ; H)$ to be the completion of the $H$-valued simple functions for the norm $\|f\|_{p}=\left\{\int|f|^{p} d \mu\right\}^{1 / p}$ where $|f|$ is the function whose values are the norms in $H$ of the corresponding values in $f$. For $L_{\infty}$ we take $H$-valued bounded measurable functions with the additional restriction that the values of the function must lie in a separable subspace of $H$. In this way we get nice functors $L_{p}(\mathfrak{Q}, \mu ; \cdot)$ of Banach spaces with dual functors $L^{p^{\prime}}(\mathfrak{Q}, \mu ; \cdot)$. In general, the elements of $L^{q}(\mathbb{Q}, \mu ; H)$ have to be regarded as $H$-valued additive set functions on $\mathfrak{Q}$. For $1<p<\infty$ we have $L^{p}(\mathbb{Q}, \mu ; H)=L_{p}(\mathbb{Q}, \mu ; H)$, "=" denotes canonical isomor- 
phism, when $H$ has the Radon-Nikodým property and $(\mathfrak{Q}, \mu)$ is $\sigma$-finite, which we are assuming. Instances of the Radon-Nikodým property are reflexive spaces and separable dual spaces. We shall use without further ado that, for $1 \leq p<\infty$, the dual Banach space to $L_{p}(\mathfrak{Q}, \mu ; H)$ is $L_{p^{\prime}}\left(\mathfrak{Q}, \mu ; H^{\prime}\right)$ whenever $H^{\prime}$ is separable or reflexive, $H^{\prime}$ being the dual of $H$. The dual pairing between $f \in L_{p}(Q, \mu ; H)$ and $\phi \in L_{p^{\prime}}\left(\mathscr{Q}, \mu ; H^{\prime}\right)$ is given by the absolutely convergent integral $\int \phi f d \mu$ where $\phi f$ is the numerical function $\langle\phi, f\rangle$ with $\langle$,$\rangle the dual pairing of H^{\prime}$ and $H$. The conditional expectation operators have natural extensions $\mathcal{E}_{m}: L_{p}(\mathbb{Q}, \mu, H) \rightarrow$ $L_{p}\left(\mathbb{Q}_{m}, \mu, H\right)$. When $1<p \leq \infty$ and $H$ is reflexive $\mathscr{E}_{m}$ may be viewed as the adjoint of the inclusion $L_{p^{\prime}}\left(\mathscr{Q}_{m}, \mu, H^{\prime}\right) \rightarrow L_{p^{\prime}}\left(\mathscr{Q}, \mu, H^{\prime}\right)$.

A particularly interesting example of a stochastic base is given this way. Let $\mathbb{Q}_{0}$ consist of the finite unions of intervals $A$ of real numbers with integral endpoints. We take the events of $\mathbb{Q}_{n}$ to be those of the form $2^{-n} A$ where $A \in \mathbb{Q}_{0}$. More generally, in $d$-dimensional space $\mathbf{R}^{d}$ take $\mathbb{Q}_{n}$ to be the ring generated by the special cubes $A$ of the form $A=\left\{x \in \mathrm{R}^{d}: k_{i} \leq 2^{n} x_{i}<k_{i}+1\right\}$ where $i=1, \ldots, d$ and each $k_{i} \in \mathbf{Z}\left(x_{i}\right.$ is the $i$ th coordinate of $\left.x\right)$. For $A \in \mathbb{Q}_{n}$ we define $\mu(A)$ to be its usual content. Let $\mathfrak{Q}$ be the completion of $\cup \mathfrak{Q}_{n}$ for the $\mu$-metric. Then $(\mathfrak{Q}, \mu)$ is the ordinary Lebesgue measure ring of $\mathbf{R}^{d}$. We call $\left\{\mathbb{Q}_{n}\right\}$ the dyadic stochastic base on $\mathrm{R}^{d}$.

In the above example we have $\tilde{E}_{m} f \rightarrow 0$ uniformly as $m \rightarrow-\infty$ for all $f \in L_{1}$. Thus we cannot have. $\int \sup _{n}\left|\mathcal{E}_{n} f\right| d \mu<\infty$ unless $\int f d \mu=0$.

In probability examples one often encounters martingales of the form $f_{n}=$ $\sum_{k=1}^{n} x_{k}$ where the $x_{k}$ are independent random variables with mean 0 . In this instance we take $\mathbb{Q}_{m}=\mathbb{Q}_{0}$ for all $m \leq 0$ where $\mathfrak{Q}_{0}$ consists of the events of probability 0 and probability 1 . For $n>0$ the $\mathbb{Q}_{n}$ correspond to coproducts of $n$ independent copies of $\mathfrak{Q}_{1}$.

2. Functions of martingale bounded mean oscillation. If $(\mathfrak{Q}, \mu)$ is a probability algebra and $\cap \mathfrak{A}_{n}$ consists only of the events of probability 0 or 1 then an element of $\mathrm{BMO}_{p}$ (with respect to the stochastic base $\left\{\mathfrak{Q}_{n}\right\}$ ) is a function $\phi \in L_{p}$ satisfying the conditions $\phi \in \mathrm{BD}$ and $\phi \in \mathrm{JN}_{p}$ given below. In the general case a function of bounded mean oscillation is not a function; rather an element $\phi \epsilon$ $\mathrm{BMO}_{p}$ is a sequence $\phi=\left\{\phi^{(m)} ; m \in \mathbf{Z}\right\}$ where each $\phi^{(m)}$ is locally in $L_{p}$, and $\mathcal{E}_{m} \phi^{(m)}=0, \phi^{(n)}=\phi^{(m)}-\mathcal{E}_{n} \phi^{(m)}$ for $n \geq m$. Thus $\phi^{(m)}$ is a replacement for $\phi-\varepsilon_{m} \phi$. The conditions that such a $\phi$ must satisfy are $\phi \in \mathrm{JN}_{p}$ and $\phi \in \mathrm{BD}$.

The Banach space $\mathrm{JN}_{p}$ consists of the sequences $\phi=\left\{\phi^{(m)}\right\}$ of locally $L_{p}$ functions, as above, for which there exists a constant $b$ such that for each $m \in \mathbb{Z}$ and each $A \in \mathbb{Q}_{m}$ we have

$$
\inf _{\psi} \int_{A}\left|\phi^{(m)}-\psi\right|^{p} d \mu \leq b^{p} \mu(A)
$$


where $\psi$ runs over the elements of $L_{p}\left(\mathfrak{Q}_{m}^{A}\right)$. The greatest lower bound of the suitable constants $b$ is the $\mathrm{JN}_{p}$ norm, notation ${ }_{p}\|\phi\|_{\infty}$. It is clear that $\phi \in \mathrm{JN}_{p}$ iff $\mathcal{E}_{m}\left\{\left|\phi^{(m)}\right|^{p}\right\}$ is uniformly bounded, and

$$
{ }_{p}\|\phi\|_{\infty} \leq \sup _{m}\left\|G_{m}\left\{\left|\phi^{(m)}\right|^{p}\right\}\right\|_{\infty}^{1 / p} \leq 2 \cdot{ }_{p}\|\phi\|_{\infty} \cdot
$$

The space $\mathrm{BD}$ consists of the sequences $\left\{\xi_{n}: n \in \mathrm{Z}\right\}$ of uniformly bounded martingale differences. That is to say, $B D$ consists of the sequences $\left\{\xi_{n}\right\}$ where $\xi_{m} \in L_{\infty}\left(Q_{m}\right), \mathcal{E}_{m-1} \xi=0$, and there exists a constant $b$ such that, for each $m$, $\inf _{\psi}\left\|\xi_{m}-\psi\right\|_{\infty} \leq b$ where $\psi$ runs over $L_{\infty}\left(\mathfrak{a}_{m-1}\right)$. The BD-norm is the greatest lower bound of the suitable constants $b$.

As a matter of notation we associate with an element $\phi=\left\{\xi_{n}\right\} \in \mathrm{BD}$ the formal sums $\phi^{(m)}=\Sigma_{n>m} \xi_{n}$. Also, given $\phi \in \mathrm{JN}_{p}$ we get a sequence of martingale differences $\left\{\xi_{n}\right\}$ defined by $\xi_{n}=\phi^{(n-1)}-\phi^{(n)}$.

The Banach space $\mathrm{BMO}_{p}, 1 \leq p<\infty$, is $\mathrm{BD} \cap \mathrm{JN}_{p}$; the norm is

$$
b_{p}(\phi)=\max \left\{\|\phi\|_{\mathrm{BD}},\|\phi\|_{\infty}\right\} \text {. }
$$

It is obvious that if $p<q$ and $\phi \in \mathrm{JN}_{q}$ then also $\phi \in \mathrm{JN}_{p}$, and $\|\phi\|_{\infty} \leq$ $q\|\phi\|_{\infty}$. For the general stochastic base $\left\{\mathfrak{Q}_{n}\right\}$ the space $\mathrm{JN}_{p}$ is strictly larger than $\mathrm{JN}_{q}$; indeed, an element $\phi \in \mathrm{JN}_{p}$ need not have the $\phi^{(m)}$ locally in $L_{q}$. By contrast we have the remarkable fact that all the $\mathrm{BMO}_{p}$ spaces are the same. An even sharper result holds.

Theorem JN. Suppose $\phi \in \mathrm{BMO}_{1}$; then

(i) $\phi \in \mathrm{BMO}_{p}$ for each $p$ and $b_{1}(\phi) \leq b_{p}(\phi)<57 p b_{1}(\phi)$,

(ii) for each integer $m$, each $A \in \mathbb{Q}_{m}$, and eacb subset $E \in \mathbb{Q}$ of $A$ we bave

$$
\int_{E}\left|\phi^{(m)}\right| d \mu<57 b_{1}(\phi) \cdot \mu(E)\{2+\log \mu(A) / \mu(E)\} \text {. }
$$

Remark. Theorem JN can be recast. With the notation $\operatorname{Pr}_{A}(E)=\mu(E \cap A) / \mu(A)$, statement (ii) gives

(ii') for each integer $m$, each $A \in \mathbb{Q}_{m}$, and each $\lambda>0$,

$$
\operatorname{Pr}_{A}\left\{\left|\phi^{(m)}\right|>\lambda\right\}<a \exp (-\lambda / b)
$$

with $a=e^{2}$ and $b=57 b_{1}(\phi)$. In turn, (ii') implies an estimate of the form (ii). It is also not hard to see that, absolute constants apart, (i) and (ii') are equivalent.

In the case of the dyadic stochastic base on $\mathrm{R}^{d}$, Theorem $\mathrm{JN}$ was first proved by John and Nirenberg [9]. They only assume $\phi \in \mathrm{JN}_{1}$, but this stochastic base has a regularity property which ensures that $b_{p}(\phi) \leq 2^{d}{ }_{p}\|\phi\|_{\infty}$, as one readily can verify.

The general statement of Theorem JN is a corollary of Theorems $\mathrm{E}$ and $\mathrm{F}_{1}$ below. Theorem $\mathrm{E}$ in $\S_{3}$ is quite straightforward, but the needed part of Theorem $\mathrm{F}_{1}$ 
uses most of the machinery of this article. Indeed, if one knew a priori the equivalence of $\mathrm{BMO}_{1}$ with $\mathrm{BMO}_{2}$ other theorems could be given quicker proofs. (Even for the dyadic stochastic base on $\mathbf{R}^{d}$, by the time one includes the John-Nirenberg proof there is no real saving over the present route.)

The distinction between functions of "ordinary" bounded mean oscillation and martingale bounded mean oscillation must be emphasized. A "function" $\phi$ on $\mathbf{R}$ belongs to ordinary $\mathrm{JN}_{1}$ if there exists a constant $b$ such that, for each interval $A, \inf _{\psi} \int_{A}|\phi-\psi| d \mu \leq b \mu(A)$, where $\psi$ runs over the constants. The space $\mathrm{JN}_{1}$ with respect to the dyadic stochastic base differs only in that the intervals $A$ of the defining condition are restricted to the class of special dyadic intervals. The odd function $\phi$ defined by $\phi(t)=\log t$ for $t>0$ is martingale BMO but not ordinary $\mathrm{BMO}$ since the $\mathrm{JN}_{1}$ condition fails for all intervals symmetric about the origin. Indeed, martingale $\mathrm{BMO}$ is not even translation invariant. In any case, the martingale BMO space is strictly larger than ordinary BMO.

The contents of this section are valid for $H$-valued functions where $H$ is an arbitrary Banach space. When $H$ is a Hilbert space the $\mathrm{JN}_{2}$-norm has the simple form

$$
{ }_{2}\|\phi\|_{\infty}=\sup _{m}\left\|\xi_{m}\left|\phi^{(m)}\right|^{2}\right\|_{\infty}^{1 / 2}=\sup _{m}\left\|\xi_{m}\left\{\sum_{k>m}\left|\xi_{k}\right|^{2}\right\}\right\|_{\infty}^{1 / 2} \cdot
$$

3. $H_{1}$-martingales. The space ${ }_{1} L_{1}$ of $L_{1}$-martingales is defined to be the subspace of $L_{1}(\mathbb{Q})$ constituted by the elements $f$ such that $\lim _{m \rightarrow-\infty}\left\|\mathcal{G}_{m} f\right\|_{1}=0$. For any $f \in L_{1}$ we have that as $n \rightarrow \infty, \mathcal{E}_{n} f \rightarrow f$ almost uniformly. In general, there is not convergence in norm. For a locally summable function $f$ we form the martingale maximal function $M f=\sup _{n}\left|\mathcal{E}_{k} f\right|$. Clearly $|f| \leq M f$. The martingale maximal theorem asserts that for $p>1$, there is the estimate $\|M f\|_{p} \leq p^{\prime}\|f\|_{p}$, and this is best possible. In general, the set of $f \in{ }_{1} L_{1}$ such that $\|M f\|_{1}<\infty$ constitutes a proper subspace. We define $H_{1}$ to be this subspace; so $H_{1}$ is a Banach space under the norm $\|f\|=\|M f\|_{1}$. (If $f$ is any measurable function such that $\|M f\|_{1}<\infty$ then $\tilde{E}_{m} f$ converges as $m \rightarrow-\infty$ in norm and almost uniformly to a function $f_{-\infty}$; we impose on $H_{1}$ the normalization condition $f_{-\infty}=0$ as an inessential convenience.)

It is a routine matter, and moreover a by-product of later results, that if $b \in H_{1}$ then $\mathcal{E}_{n} b-\mathcal{E}_{m} b$ converges to $b$ in norm as $m \rightarrow-\infty, n \rightarrow+\infty$. The point of this remark is that it is sufficient to check certain inequalities on martingales $f$ such that $\varepsilon_{k} f=0$ for $k<k_{0}$ and $\tilde{E}_{k} f=\varepsilon_{l} f$ for $k \geq l$ where $k_{0}$ and $l$ are arbitrarily given integers. In particular, the space AM of absolutely convergent martingales constitutes a dense subset of $H_{1}$. The elements $a \in \mathrm{AM}$ are the martingales $a=$ $\Sigma z_{n}$ where $z_{n} \in L_{1}\left(\mathscr{Q}_{n}\right), \tilde{\sigma}_{n-1} z_{n}=0$, and $\|a\|=\Sigma\left\|z_{n}\right\|_{1}<\infty$. 
A central fact about $H_{1}$ is that its dual Banach space is equivalent to BMO. The precise statement is Theorem EF which we split as

Theorem E. Let $\Phi$ be a bounded linear functional on $H_{1}$, and put $\|\Phi\|=$ $\sup |\Phi(b)| /\|M b\|_{1}$ for $b \in H_{1}$. Then $\Phi$ bas the form $\Phi(b)=\int \phi b d \mu$ where

(i) $\phi \in \mathrm{BMO}_{p}$ for each $p$ and $b_{p}(\phi) \leq p\|\Phi\|$;

(ii) for eacb integer $m$, each $A \in \mathbb{Q}_{m}$, and each subset $E \in \mathbb{Q}$ of $A$ we have $\int_{E}\left|\phi^{(m)}\right| d \mu \leq\|\Phi\| \mu(E)\{2+\log \mu(A) / \mu(E)\}$.

Theorem $\mathbf{F}_{1}$. Suppose $\phi \in \mathrm{BMO}_{1}$. Then $b \rightarrow \int \phi b d \mu$ gives a continuous linear functional on $H_{1}$ with $\left|\int b \phi d \mu\right|<57 b_{1}(\phi)\|M b\|_{1}$.

(Weaker versions of this result with $\mathrm{BMO}_{1}$ replaced by $\mathrm{BMO}_{p}$ are designated as "Theorem $\mathrm{F}_{p}$ ".)

The combined result, Theorem EF, is simply a dual version of the basic Theorem $B$. Theorem $\mathrm{E}$ is, however, so easy to prove directly that we do so. First we observe the obvious

Proposition 1. The dual Banach space to $\mathrm{AM}$ is $\mathrm{BD}$.

The duality pairing of $\mathrm{AM}$ and $\mathrm{BD}$ is given by $\int \phi b d \mu=\Sigma_{n} \int \xi_{n} y_{n} d \mu$, an absolutely convergent sum of absolutely convergent integrals when $b=\Sigma y_{n} \in A M$ and $\phi=\left\{\xi_{n}\right\} \in \mathrm{BD}$. Since $\mathrm{AM}$ is dense in $H_{1}$ any linear functional $\Phi \in \mathrm{H}_{1}^{n}$ is determined by a unique $\phi \in \mathrm{BD}$. For each pair of integers $m>n$ we can write down the finite sum $\mathcal{E}_{n} \phi^{(m)}=\Sigma_{k=m+1}^{n} \xi_{k}$. Then for any $b \in H_{1}$ with $\tilde{E}_{m} b=0$ we shall have

$$
\Phi(b)=\lim _{n \rightarrow \infty} \Phi\left(\tilde{E}_{n} b\right)=\lim _{n \rightarrow \infty} \int\left(\mathcal{E}_{n} \phi^{(m)}\right) b d \mu .
$$

Now suppose we have an event $A \in \mathbb{Q}_{m}$ and a function $\theta \in L \log L\left(\mathbb{Q}^{A}\right)$. Then the function $b=\theta-\mathcal{E}_{m} \theta$ belongs to $H_{1}$ and $\Phi\left(\theta-\mathcal{E}_{m} \theta\right)=\int_{A} \phi^{(m)} \theta d \mu$ where $\phi^{(m)}=\lim _{n \rightarrow \infty} \mathcal{E}_{n} \phi^{(m)}$, the limit necessarily existing in the norm of $L_{q}^{(m)}\left(Q^{A}\right)$ for all $q<\infty$. The statement (i) of Theorem E says $\|\phi\|_{\mathrm{BD}} \leq\|\Phi\|$, which we know from Proposition 1, and ${ }_{p}\|\phi\|_{\infty} \leq p\|\Phi\|$ which follows by taking suitable $\theta \in L_{p^{\prime}}$. We omit the details which are similar to those of the proof of (ii) given next (moreover, apart from a constant factor (ii) is equivalent to ${ }_{p}\|\phi\|_{\infty} \leq p\|\Phi\|$ for all $p$ ). Given $A \in \mathbb{Q}_{m}$ and $E \subset A$ we observe that

$$
\int_{E}\left|\phi^{(m)}\right| d \mu=\sup _{\theta}\left|\int_{A} \phi^{(m)} \theta d \mu\right|=\sup _{\theta}\left|\Phi\left(\theta-\xi_{m} \theta\right)\right|
$$

where $\theta$ ranges over the measurable functions with $|\theta| \leq E$. The $H_{1}$-norm of $b=$ $\theta-\mathcal{E}_{m} \theta$ for such a $\theta$ is estimated by

$$
\|M b\|=\int \sup _{k \geq m}\left|E_{k} \theta-\mathcal{E}_{m} \theta\right| d \mu \leq \int_{A} M \theta d \mu+\int_{A}\left|\mathcal{E}_{m} \theta\right| d \mu \leq \int_{A} M E d \mu+\int_{A} E d \mu
$$


since $\left|\delta_{k} \theta\right| \leq \tilde{E}_{k} E$. Now $\int_{A} E d \mu=\mu(E)$, and the maximal inequality gives

$$
\int_{A} M E d \mu \leq \mu(E)\{1+\log \mu(A) / \mu(E)\} \text {. }
$$

The above extends to vector-valued martingales. For any Banach space $H$ we define $H_{1}(H)$ taking $H$-valued functions $f \in{ }_{1} L_{1}(H)$ with $\|M f\|_{1}<\infty$. Theorem $F_{1}$ is valid with $\phi \in \mathrm{BMO}_{1}\left(H^{\prime}\right)$ where $H^{\prime}$ is the dual space of $H$. In Proposition 1 the duality is between $\mathrm{AM}(H)$ and $\mathrm{BD}\left(H^{\prime}\right)$; in Theorem $\mathrm{E}$ the spaces $H_{1}(H)$ and $\mathrm{BMO}_{p}\left(H^{\prime}\right)$ are involved; for the validity of these assertions we assume that $H^{\prime}$ is separable or reflexive.

4. Martingale contractions. Let $(\mathfrak{A}, \mu)$ be a measure ring and $H$ and $\mathcal{H}$ a pair of Hilbert spaces. We consider à bounded linear transformation $V: L_{2}(\mathscr{Q}, \mu ; H) \rightarrow$ $L_{2}(\mathfrak{Q}, \mu ; \mathcal{H})$ which for simplicity we suppose has bound $\leq 1$. The transformation $V$ will be called a "martingale contraction" with respect to the stochastic base $\left\{\mathfrak{Q}_{n}: n \in \mathbf{Z}\right\}$ for $(\mathfrak{Q}, \mu)$ if three further conditions are satisfied:

Basic condition. $V$ commutes with all the conditional expectation operators $\tilde{E}_{n}, n \in \mathrm{Z}$.

Measurability condition. Given an integer $m$, an event $A \in \mathbb{Q}_{m}$, and a function $f \in L_{2}^{(m)}(\mathbb{Q}, \mu ; H)$ we have $V(A f)=A(V f)$.

Norm condition. For martingale differences $x_{n} \in L_{1}^{(n-1)} \cap L_{2}\left(\mathscr{Q}_{n}, \mu ; H\right)$ we have $\left\|V x_{n}\right\|_{1} \leq\left\|x_{n}\right\|_{1}$. (It is not needed here, but it is natural to assume that the adjoint $V^{\prime}$ also satisfies the norm condition, equivalently, if $\phi \in \mathrm{BD} \cap L_{2}$ then $\left.\|V \phi\|_{\mathrm{BD}} \leq\|\phi\|_{\mathrm{BD}} \cdot\right)$

As will soon be clear, it is essential to consider martingale contractions in the generality stated. We intend to deal more fully with them elsewhere, but a typical result is a weak $L_{1}$-estimate for maximal martingale contractions $M V f=$ $\sup _{k}\left|\mathcal{E}_{k} V f\right|, \mu\{M V f \geq \lambda\}<5 \lambda^{-1}\|f\|_{1}$ leading to $L_{p}$ bounds for $1<p<\infty$, of the form $\|M V f\|_{p}<10 p p^{\prime}\|f\|_{p}$. Such results are essentially due to D. G. Austin [1] and to Burkholder and Gundy [2]. In general $V$ will not be bounded on $L_{1}$.

The basic result in the $H_{1}$-theory of martingale contractions is

Theorem C. Martingale contractions give bounded linear transformations of $H_{1}$-spaces. If $b \in H_{1}$ and $V$ is a martingale contraction then $\|M V b\|_{1}<30\|M b\|_{1}$ where $M b$ is the maximal function of $b$ and $M V b$ the maximal function of $V b$.

Theorem $C$ is an immediate consequence of Theorem $B_{2}$ (a weaker special case of Theorem B). We defer the proof until after the statement and proof of Theorem B in $\S 8$ below.

A corollary of Theorem C is the theorem of Burgess Davis [3].

Theorem D. Given a martingale $f$ define $|\Gamma f|$ by $|\Gamma /|^{2}=\Sigma\left|x_{n}\right|^{2}$ where $x_{n}=$ $\mathcal{E}_{n} f-\mathcal{E}_{n-1} f$. Then we bave the inequalities 


$$
\|\Gamma f\|_{1}<30\|M f\|_{1}, \quad\|M f\|_{1}<11\|\Gamma f\|_{1} \cdot
$$

Proof. We suppose $f$ is a martingale with values in a Hilbert space $H$ and we define a new martingale $\Gamma f$, with values in $l_{2}(H)$, by $\Gamma f=\left\{x_{n}\right\}$. Here $M \Gamma f=$ $|\Gamma f|$ since $\left|E_{n} \Gamma f\right|=\left\{\Sigma_{k \leq n}\left|x_{k}\right|^{2}\right\}^{1 / 2}$ is increasing. Now $f \rightarrow \Gamma f$ is a martingale contraction, as is its adjoint. Applying Theorem $C$ to $b=f, V=\Gamma$ we get $\|\Gamma b\|_{1}<30\|M b\|_{1}$; applying it to $b=\Gamma f$ and $V=\Gamma^{\prime}$ (observe that $\Gamma^{\prime} \Gamma f=f$ ) we get $\|M b\|_{1}<30\|\Gamma b\|_{1}$. A slight modification of detail allows for a better estimate.

A proof of Theorem D by methods similar to ours is given by Garsia [6].

Theorem $C$ suggests the following idea. Let us say that $\tilde{f}$ is a conjugate martingale to $f$ if $\tilde{f}=c V f$ and $f=d W \tilde{f}$ where $V$ and $W$ are martingale contractions and $c$ and $d$ are constants. Then for each $p, 1 \leq p<\infty$, the following are equivalent:

(i) $M f \in L_{p}$,

(ii) $M \tilde{f} \in L_{p}$ for all conjugate functions $\tilde{f}$,

(iii) $M \widetilde{T} \in L_{p}$ for some conjugate function $\tilde{T}$,

(iv) $\tilde{f} \in L_{p}$ for all conjugate functions $\tilde{f}$,

(v) $\widetilde{T} \in L_{p}^{p}$ for the particular conjugate function $\widetilde{T}=\Gamma f$.

Any of these five equivalent conditions can serve to define the Banach space $H_{p}$. For $p>1$, the space $H_{p}$ is equivalent to $L_{p}$, and so it gives nothing new. We have chosen (i) to define $H_{1}$. Condition (iv) suggests function-theoretic analogues where the role of martingale contraction is replaced by CalderónZygmund operator, see [5].

5. $L_{p}$-regulated $L_{1}$-martingales. We are going to introduce spaces ${ }_{p} L_{1}, 1 \leq$ $p \leq \infty$, of martingales which have a probabilistic interest beyond the role they play here. The definition of ${ }_{p} L_{1}$ is made in such a way that the properties of $p_{1} L_{1}$-martingales are easy to establish. The main theorems give useful characterizations of ${ }_{p} L_{1}$ when $p=\infty$ and $p=2$; the case $p=1$ is trivial since ${ }_{1} L_{1}$ consists precisely of the $L_{1}$-martingales.

The idea of an $L_{p}$-regulated $L_{1}$-martingale is most easily seen in a special case. Suppose $f=g \psi$ where $g \in L_{p^{\prime}}\left(\mathfrak{Q}_{m}\right)$ and $\psi \in L_{p}^{(m)}(\mathfrak{Q})$, the subspace of $L_{p}(\mathfrak{Q})$ consisting of elements with $\tilde{E}_{m} \psi=0$. For such an $f$ we have $\tilde{E}_{k} f=$ $g \tilde{G}_{k} \psi$; moreover if $V$ is any martingale contraction then $V f=g V \psi$. These special martingales are clearly very nice, but to obtain a Banach space we have to allow certain infinite sums. To be exact, ${ }_{p} L_{1}$ consists of the $L_{1}$-martingales $/$ which can be written in the form $f=\Sigma g \psi$ where $\Sigma\|g\|_{p^{\prime}}\|\psi\|_{p}<\infty$. The ${ }_{p} L_{1}$ norm is the infimum of the $\Sigma\|g\|_{p^{\prime}}\|\psi\|_{p}$ for all possible representations.

For $f \in{ }_{\infty} L_{1}$ an especially simple representation is possible. Given $\epsilon>0$ we can write $f=\Sigma f_{A}$ with $\Sigma \mu(A)\left\|f_{A}\right\|_{\infty}<{ }_{\infty}\|f\|_{1}+\epsilon$, a countable sum over events 
$A$ where for some $m=m(A)$, we have $A \in \mathbb{Q}_{m}$ and $f_{A}$ is a bounded measurable function vanishing outside $A$ with $\mathscr{E}_{m} f_{A}=0$.

The connection between the ${ }_{p} L_{1}$ spaces and something which we have already seen is given by

Proposition 2. For $1<p<\infty$ the dual Banach space to ${ }_{p} L_{1}$ is $\mathrm{JN}_{p^{\prime}}$. The space $\mathrm{JN}_{1}$ may be identified with a closed subspace of the dual of ${ }_{\infty} L_{1}$.

Proposition 2 results from abstract nonsense. It goes beyond what we really need, which is the estimate

$$
\left|\int \phi f d \mu\right| \leq{ }_{p}\|\phi\|_{\infty} \cdot{ }_{p}\|f\|_{1} \text { for } f \epsilon_{p_{1}} L_{1} \text { and } \phi \in \mathrm{JN}_{p^{\prime}}, 1<p \leq \infty .
$$

This estimate is rather concrete. If $f=g \psi$ where $g \in L_{p^{\prime}}\left(\mathfrak{Q}_{m}\right)$ and $\psi \in L_{p}^{(m)}(\mathfrak{Q})$ then for $\phi \in \mathrm{JN}_{p^{\prime}}$ the function $\mathcal{E}_{m}\left(\phi^{(m)} \psi\right)$ belongs to $L_{p}\left(\mathfrak{Q}_{m}\right)$ and $\left\|\mathcal{E}_{m}\left(\phi^{(m)} \psi\right)\right\|_{p}$ $\leq_{p^{\prime}}\|\phi\|_{\infty}\|\psi\|_{p}$. Thus $\int \phi f d \mu$ with $f$ of the special form is defined by the absolutely convergent integral $\int g \mathcal{G}_{m}\left(\phi^{(m)} \psi\right) d \mu$. The definition of $\int \phi f d \mu$ is extended to all $f \epsilon_{p} L_{1}$ by linearity and continuity, and the estimate $(*)$ obviously persists.

The proof of Proposition 2 results from a precise description of ${ }_{p} L_{1}$. For fixed $p$ and each $m \in \mathbf{Z}$ we form $\Lambda_{m}=L_{p^{\prime}}\left(\mathbb{Q}_{m}\right) \otimes_{\mathbb{Q}_{m}} L_{p}^{(m)}(\mathbb{Q})$, where $\otimes_{\mathbb{Q}_{m}}$ is the quotient of the Banach space tensor product by the subspace generated by the elements of the form $A_{g} \otimes \psi-g \otimes A \psi$ where $A \in \mathbb{Q}_{m}$. Next let $\Lambda$ be the Banach space sum, $\Lambda=\Sigma_{m} \Lambda_{m}$. For each $m$ there is a morphism $P_{m}: \Lambda_{m} \rightarrow{ }_{1} L_{1}$ given by $P_{m}(g \otimes \psi)=g \psi$. Then $P=\Sigma_{m} P_{m}$ gives a morphism $P: \Lambda \rightarrow{ }_{1} L_{1}$ whose coimage, i.e. $\Lambda /$ kernel $P$ considered as identified with a subset of ${ }_{1} L_{1}$, is precisely ${ }_{p} L_{1}$.

When $H$ is a Banach space, the space ${ }_{p} L_{1}(H)$ of $H$-valued $L_{p}$-regulated $L_{1}$ martingales is defined by taking $\Lambda_{m}(H)=L_{p^{\prime}}\left(\mathscr{Q}_{m} ; \mathbf{R}\right) \otimes_{Q_{m}} L_{p}^{(m)}\left(\mathscr{Q}_{;} ; H\right)$ and then proceeding in exactly analogous fashion.

If $\phi \in \mathrm{JN}_{p^{\prime}}\left(H^{\prime}\right)$ and $f \in{ }_{p} L_{1}(H)$ then (*) persists, but in order to ensure that $\mathrm{JN}_{p^{\prime}}\left(H^{\prime}\right)$ is the full dual space of ${ }_{p} L_{1}(H)$ when $1<p<\infty$ we assume that $H^{\prime}$ is separable or reflexive.

I defer the details of the proof of Proposition 2 until $\$ 9$. All that is involved is a pedantic recasting of the definition of $\mathrm{JN}_{p}$ combined with purely categorical arguments; no analysis enters.

I have included the pedantic definition of ${ }_{p} L_{1}$ here to point out to those with any familiarity with tensor products that, while the formal definition of ${ }_{p} L_{1}$ is very convenient, a useful description of these spaces would not ordinarily be expected. A happy surprise is in store. Read on.

6. $L_{\infty}$-regulated $L_{1}$-martingales. The first of our two main results is a simple characterization of $\infty_{\infty} L_{1}$-martingales. 
Theorem $A_{\infty}$. If $f \in \epsilon_{\infty} L_{1}$ then there exists an increasing sequence $\left\{r_{n}\right\}$ with $r_{n} \in L_{1}^{+}\left(\mathscr{Q}_{n}\right)$ and each $\int r_{n} d \mu \leq_{\infty}\|f\|_{1}$ sucb that $\left|\xi_{n+1} f\right| \leq r_{n}$. Conversely, if $f$ is a martingale such that $\left|\mathcal{E}_{n+1} f\right| \leq r_{n}$ where $\left\{r_{n}\right\}$ is an increasing sequence of functions $r_{n} \in L_{1}^{+}\left(\mathscr{Q}_{n}\right)$ with $\int r_{n} d \mu \leq \rho$, then $f \in \epsilon_{\infty} L_{1}$ and ${ }_{\infty}\|f\|_{1}<6 \rho$.

The first part of the statement is completely straightforward. Suppose $f=g \psi$ where $g \in L_{1}^{+}\left(\mathfrak{Q}_{m}\right), \psi \in L_{\infty}(\mathfrak{Q})$ and $\mathscr{G}_{m} \psi=0$. Put $r_{n}=0$ for $n<m$ and $r_{n}=$ $\|\psi\|_{\infty} g$ for $n>m$. Then all the conditions are satisfied since $\mathcal{E}_{n+1} f=0$ when $n<m$ and $\mathcal{E}_{n+1} f=g \mathcal{G}_{n+1} \psi$ when $n \geq m$, and $\left\|\mathcal{G}_{n+1} \psi\right\|_{\infty} \leq\|\psi\|_{\infty}$. The result extends from $f$ of the special form to all $f \epsilon_{\infty} L_{1}$.

The nonobvious part of the statement is the converse result. We shall use a stopping-time argument. Choose a number $c>1$, and define a stopping time $r_{i}$ by $\boldsymbol{r}_{i}=$ first $n \in \mathrm{Z}$ such that $r_{n}>c^{i}$. We put $f^{i}$ for the martingale $f$ stopped according to $r_{i}$ and $B^{i}=\left\{\tau_{i}<\tau_{i+1}\right\}$. Then we may write $f=\sum B^{i}\left(f^{i+1}-f^{i}\right)$. What this means is that for each $i$ we have sequences of events

$$
A_{k}^{i}=\left\{r_{k} \leq c^{i}\right\} \in \widetilde{\mathbb{Q}}_{k}, \quad B_{k}^{i}=\left\{r_{k-1} \leq c^{i}<r_{k} \leq c^{i+1}\right\} \in \widetilde{\mathbb{Q}}
$$

in terms of which we have

$$
f^{i}=\sum_{k} A_{k}^{i}\left(\mathcal{E}_{k+1} f-\mathcal{E}_{k} f\right), \quad B^{i}=\bigcup_{k} B_{k}^{i} .
$$

Now let us fix temporarily $m$ and $i$ and examine the term $B_{m}^{i}\left(f^{i+1}-f^{\dot{i}}\right)$ which we write as $g \psi$ where

$$
g=B_{m}^{i} c^{i+1} \text { and } \psi=B_{m}^{i} c^{-i-1}\left(f^{i+1}-f^{i}\right) .
$$

We wish to establish two facts:

$$
\|\psi\|_{\infty} \leq 1+c^{-1} \text { and } \xi_{m} \psi=0 .
$$

In the event $B_{m}^{i}$ we have $f^{i}=\mathcal{E}_{m} f$, and we know, by hypothesis, that $\left|\mathcal{E}_{m} f\right| \leq$ $r_{m-1}$. Thus $B_{m}^{i}\left|f^{i}\right| \leq c^{i}$, and it suffices to show that $\left|f^{i+1}\right| \leq c^{i+1}$ and $\xi_{m} f^{i+1}=$ $\xi_{m} f$ in the event $B_{m}^{i}$. Recall the definition of $f^{i+1}$,

$$
\begin{array}{ll}
f^{i+1}=\mathcal{E}_{n} f & \text { in the event }\left\{r_{n-1} \leq c^{i+1}<r_{n}\right\}, \\
f^{i+1}=f \quad \text { in the event }\left\{\sup _{k} r_{k} \leq c^{i+1}\right\} .
\end{array}
$$

Therefore $\left|f^{i+1}\right| \leq c^{i+1}$, and in $B_{m}^{i}$ we have

$$
f^{i+1}=\mathcal{E}_{m} f+\sum_{k \geq m} A_{k}^{i+1}\left(\mathcal{E}_{k+1} f-\mathcal{E}_{k} f\right)
$$

Since $\mathcal{E}_{m}=\mathcal{E}_{m} \mathcal{E}_{k}$ whenever $k \geq m$, the operator $\mathcal{E}_{m}$ annihilates each term in the sum, and we have indeed $\mathcal{E}_{m} f^{i+1}=\mathcal{E}_{m} f$ in $B_{m}^{i}$. 
What we have shown so far is that $B_{m}^{i}\left(f^{i+1}-f^{i}\right)=g \psi$ where $g \in L_{1}\left(Q_{m}\right)$ and $\psi \in L_{\infty}^{(m)}(\mathfrak{Q})$. Thus $B_{m}^{i}\left(f^{i+1}-f^{i}\right)$ is an element of $\infty_{\infty} L_{1}$ and ${ }_{\infty}\left\|B_{m}^{i}\left(f^{i+1}-f^{i}\right)\right\|_{1} \leq$ $\|g\|_{1}\|\psi\|_{\infty} \leq(c+1) c^{i} \mu\left(B_{m}^{i}\right)$. It follows that ${ }_{\infty}\|f\|_{1} \leq(c+1) \int \beta d \mu$ where $\beta=$ $\Sigma B_{m}^{i} c^{i}$, the sum being taken over all $m$ and $i$.

Let $j$ be the random variable, whose value is an integer or $+\infty$, defined by $j=\sup \left\{i: c^{i}<r\right\}$. Then we have the pointwise estimate

$$
\beta \leq \sum_{i \leq j} c^{i} \leq c^{j} \sum_{k=0} c^{-k}=c^{j+1}(c-1)^{-1} \leq c(c-1)^{-1} r .
$$

This gives the norm estimate ${ }_{\infty}\|f\|_{1} \leq c(c+1)(c-1)^{-1} \rho$. The choice $c=2$ gives the assertion of the theorem; the best choice is $c=1+\sqrt{ } 2$.

Theorem $A_{\infty}$ remains valid for martingales with values in an arbitrary Banach space.

7. The space of ${ }_{2} L_{1}$-martingales. Given a martingale $f$ we form a LittlewoodPaley function $\gamma(f)$ defined by

$$
\gamma^{2}(f)=\sum_{n} \varepsilon_{n-1}\left|x_{n}\right|^{2}, \quad x_{n}=\varepsilon_{n} f-\varepsilon_{n-1} f
$$

The function $\gamma(f)$ has played a fundamental role in the study of martingales since the work of P. Lévy (see [10, Chapter VIII] where the notation is $b_{n}$ for $\gamma_{n}(f)$ ). There is a remarkable characterization of ${ }_{2} L_{1}$ in terms of $\gamma$.

Theorem $\mathrm{A}_{2}$. A necessary and sufficient condition that $f$ belong to ${ }_{2} L_{1}$ is that $\gamma(f)$ be summable. More precisely we have $\int \gamma(f) d \mu \leq_{2}\|f\|_{1} \leq 2^{1 / 2} \int \gamma(f) d \mu$.

Proof. Consider $f \epsilon_{2} L_{1}$ of the form $f=g \psi$ where $g \in L_{2}^{+}\left(\mathbb{Q}_{m}\right)$ and $\psi \epsilon$ $L_{2}^{(m)}(\mathfrak{Q})$. Then we have $\gamma(f)=g \gamma(\psi)$, but $\|\gamma(\psi)\|_{2}=\|\psi\|_{2}$. Hence $\|\gamma(f)\|_{1} \leq$ $\|g\|_{2}\|\gamma(\psi)\|_{2}=\|g\|_{2}\|\psi\|_{2}={ }_{2}\|f\|_{1}$, and this inequality obviously persists for all $f \in{ }_{2} L_{1}$. In the opposite direction it suffices to prove that for $\phi \epsilon \mathrm{JN}_{2}$, the dual space of ${ }_{2} L_{1}$ we have $|\langle f, \phi\rangle| \leq 2^{1 / 2}\|\gamma(f)\|_{1} \cdot{ }_{2}\|\phi\|_{\infty}$. We can write $\langle f, \phi\rangle=$ $\int \Sigma x_{k} \xi_{k} d \mu$ where $x_{k}=\xi_{k} f-\xi_{k-1} f, \xi_{k}=\phi^{(k-1)}-\phi^{(k)}$. The Schwartz inequality gives $|\langle f, \phi\rangle| \leq \alpha \beta$ where

$$
a^{2}=\int \sum \gamma_{n}^{-1}\left|x_{n+1}\right|^{2} d \mu, \quad \beta^{2}=\int \sum \gamma_{n}\left|\xi_{n+1}\right|^{2} d \mu
$$

and $\left\{\gamma_{n}\right\}$ is any convenient sequence. We take $\gamma_{n}=\gamma_{n}(f)=\left\{\Sigma_{k \leq n} \delta_{k}\left|x_{k+1}\right|^{2}\right\}^{1 / 2}$. Now for the computation of $\alpha$ we have $\alpha^{2}=\int \Sigma \gamma_{n}^{-1}\left(\gamma_{n}^{2}-\gamma_{n-1}^{2}\right) d \mu$ since $\int \gamma_{n}^{-1}\left|x_{n+1}\right|^{2} d \mu=\int \gamma_{n}^{-1} \xi_{n}\left|x_{n+1}\right|^{2} d \mu$. On the other hand $\Sigma \gamma_{n}^{-1}\left(\gamma_{n}^{2}-\gamma_{n-1}^{2}\right) \leq 2 \gamma$ for any increasing sequence $\left\{\gamma_{n}\right\}$ of positive quantities with $\lim _{n \rightarrow \infty} \gamma_{n}=\gamma$ (compare the sum with $\int_{-\infty}^{\infty} F^{-1 / 2}(t) d F(t)$ where $F$ is a nice increasing function with $\left.F(n)=\gamma_{n}^{2}\right)$. Hence we get $a^{2} \leq 2\|\gamma\|_{1}$. To estimate $\beta$ we observe that 
$\beta^{2}=\int \Sigma\left(\gamma_{n}-\gamma_{n-1}\right)\left|\phi^{(n)}\right|^{2} d \mu$ since $\phi^{(n)} \sim \Sigma_{k>n} \xi_{k}$. Inside the integral we may replace $\left|\phi^{(n)}\right|^{2}$ by $\varepsilon_{n}\left|\phi^{(n)}\right|^{2}$, a quantity majorized by ${ }_{2}\|\phi\|_{\infty}^{2}$. Thus $\beta^{2} \leq$ $\|\gamma\|_{1} \cdot{ }_{2}\|\phi\|_{\infty}^{2}$, and the required estimate follows.

There is a somewhat more precise version of Theorem $A_{2}$ which shows more of a resemblance to Theorem $A_{\infty}$, namely

$$
{ }_{2}\|f\|_{1}=\inf \rho^{1 / 2} \cdot\left\{\int \sum_{n} r_{n}^{-1}\left|x_{n+1}\right|^{2} d \mu\right\}^{1 / 2}
$$

where the infimum is taken over all increasing sequences $\left\{r_{n}\right\}$ with $r_{n} \in L_{1}^{+}\left(\mathbb{Q}_{n}\right)$ and $\rho=\sup _{n}\left\|r_{n}\right\|_{1}<\infty$.

To prove the last statement one shows that the dual space of ${ }_{2} L_{1}$ defined the new way is again $\mathrm{JN}_{2}$.

All of the above is valid for martingales with values in a Hilbert space. It does not extend to martingales with values in an arbitrary Banach space since we have used heavily the fact that martingale differences form an orthogonal sequence of functions.

8. Representation theorem for $H_{1}$-martingales. The key result on $H_{1}$-martingales is the next

Theorem B. Suppose $1<p \leq \infty$. Given an absolutely convergent martingale $a \in \mathrm{AM}$ and an $L_{p}$-regulated $L_{1}$-martingale $f \epsilon_{p} L_{1}$ then $b=a+f$ is an $H_{1}$-martingale with $\|M b\|_{1} \leq\|a\|+p^{\prime} \cdot{ }_{p}\|f\|_{1}$. Conversely any $b \in H_{1}$ can be written in the form $b=a+f$ with $a \in \mathrm{AM}, f \in{ }_{p} L_{1}$ and $\|a\| \leq 4\|M b\|_{1},{ }_{p}\|f\|_{1}<53\|M b\|_{1}$.

Proof. It is obvious that if $a \in A M$ then $\|M a\|_{1} \leq\|a\|$. Suppose $f \epsilon_{p} L_{1}$ has the form $f=g \psi$ with $g \in L_{p^{\prime}}\left(\mathfrak{Q}_{m}\right)$ and $\psi \in L_{p}^{(m)}(\mathfrak{Q})$. Then the maximal function of $f$ is $M f=|g| M \psi$. According to the maximal inequality, $\|M \psi\|_{p} \leq p^{\prime}\|\psi\|_{p}$. Thus

$$
\|M f\|_{1} \leq\|g\|_{p^{\prime}}\|M \psi\|_{p} \leq p^{\prime}\|g\|_{p^{\prime}}\|\psi\|_{p}=p^{\prime} \cdot{ }_{p}\|f\|_{1},
$$

and this estimate extends to all $f \epsilon_{p} L_{1}$. The converse is not obvious and uses, with a trivial modification, the Burgess Davis decomposition [3] followed by an application of Theorem $A_{\infty}$. Given $b \in{ }_{1} L_{1}$ with $\|M b\|_{1}<\infty$, put $y_{n}=\varepsilon_{n} b-\varepsilon_{n-1} b$ and $A_{n} \in \mathbb{Q}_{n}$ the event $\left\{\left|y_{n}\right|>4 M_{n-1} b\right\}$. We decompose $b$ as $b=a+f$ where $a=$ $\Sigma z_{n}, f_{n}=b_{n-1}-a_{n-1}+x_{n}$ where $b_{k}=E_{k} h, a_{k}=E_{k} a$ and $z_{k}=A_{k} y_{k}-E_{k-1}\left(A_{k} y_{k}\right)$, $x_{k}=\left(1-A_{k}\right) y_{k}+\mathcal{E}_{k-1}\left(A_{k} y_{k}\right)$. We always have $\left|y_{n}\right| \leq M_{n} b+M_{n-1} b$, and in the event $A_{n}$ there is the estimate $\left|y_{n}\right|>4 M_{n-1} b$. Therefore $A_{n}\left|y_{n}\right| \leq 2\left(M_{n} b-M_{n-1} b\right)$. Now $\left\|z_{n}\right\|_{1} \leq 2\left\|A_{n} y_{n}\right\|_{1}$, so we obtain

$$
\|a\|=\sum\left\|z_{n}\right\|_{1} \leq 4 \sum_{n} \int\left(M_{n} b-M_{n-1} b\right) d \mu=4\|\cdot M b\|_{1} .
$$


For the martingale $f$ determined by $\mathcal{E}_{n} f=f_{n}$ we have $\left|f_{n}\right| \leq\left|b_{n-1}\right|+\Sigma_{k<n} A_{k}\left|y_{k}\right|+$ $\Sigma_{k<n} \mathcal{E}_{k-1} A_{k}\left|y_{k}\right|+\left(1-A_{n}\right)\left|y_{n}\right|+\mathcal{E}_{n-1} A_{n}\left|y_{n}\right|$. Using the fact that $\left(1-A_{n}\right)\left|y_{n}\right| \leq$ $4 M_{n-1} b$, we get the estimate $\left|f_{n}\right| \leq r_{n-1}$ where $r_{n-1}=5 M_{n-1} b+\Sigma_{k<n} A_{k}\left|y_{k}\right|+$ $\Sigma_{k \leq n} \mathbb{E}_{k-1} A_{k}\left|y_{k}\right|$. The crucial observation is that $r_{m} \in L^{+}\left(\mathscr{Q}_{m}\right)$. Also

$$
\left\|r_{m}\right\|_{1} \leq 5\|M b\|_{1}+2 \sum_{k}\left\|A_{k} y_{k}\right\|_{1} \leq 9\|M b\|_{1} \text {. }
$$

We now invoke Theorem $A_{\infty}$ which yields

$$
{ }_{\infty}\|f\|_{i} \leq(3+2 \sqrt{ } 2)\|r\|_{1}<53\|M b\|_{1} \text {. }
$$

The estimate for $p=\infty$ holds a fortiori for all values of $p$.

Theorem B for a particular choice of $p$ will be termed Theorem $\mathrm{B}_{p}$. The hardest case is $\mathrm{B}_{\infty}$. When $p=2$ we can prove that ${ }_{2}\|f\|_{1} \leq 2$ sup $\left\|r_{m}\right\|_{1}$ in the above construction by using Theorem $A_{2}$. Thus Theorem $B_{2}$ with the estimate ${ }_{2}\|f\|_{1}<$ $13\|M b\|_{1}$ bypasses Theorem $A_{\infty}$, at least for Hilbert space-valued martingales. Now suppose $V: L_{2}(\mathfrak{Q}, \mu ; H) \rightarrow L_{2}(\mathfrak{Q}, \mu ; \mathcal{H})$ is a martingale contraction and $f \epsilon$ ${ }_{2} L_{1}(\mathfrak{Q}, \mu ; H)$. We assert that $V f \epsilon_{2} L_{1}(\mathbb{Q}, \mu ; \mathcal{H})$ and ${ }_{2}\|V f\|_{1} \leq_{2}\|f\|_{1}$. It is enough to consider $f$ of the special form $f=g \psi$ where $g \in L_{2}\left(\mathbb{Q}_{m} ; \mathbf{R}\right)$ and $\psi \in L_{2}^{(m)}\left(\mathbb{Q}_{;} H\right)$. We know that $\|V \psi\|_{2} \leq\|\psi\|_{2}$ and $\mathcal{E}_{m} V \psi=V \mathcal{E}_{m} \psi=0$ from the basic condition. Also $V(g \psi)=g(V \psi)$ from the measurability condition. Hence

$$
{ }_{2}\|V f\|_{1} \leq\|g\|_{2}\|V \psi\|_{2} \leq\|g\|_{2}\|\psi\|_{2}={ }_{2}\|f\|_{1} .
$$

The norm condition for martingale contractions says precisely that we have a morphism of AM spaces. Thus Theorem $C$ follows from Theorem $B_{2}$.

In view of Propositions 1 and 2, Theorem EF is a dual version of Theorem B. The difficult point, Theorem $F_{1}$, involves the deep part of Theorem $B$. Theorem $B_{2}$ gives only Theorem $F_{2}$ as a corollary. It should be observed that Theorem $B$ is valid for martingales with values in any Banach space. in $\S 2$.

9. The spaces $\mathrm{JN}_{p}$. We give here a more abstract definition of $\mathrm{JN}_{p}$ than that

Fix a value of $p$ and for each $m \in \mathbf{Z}$ consider the Banach space $\Lambda_{m}^{\prime}$ defined by

$$
\Lambda_{m}^{\prime}=\operatorname{HOM}^{\mathbb{Q}_{m}}\left(L_{p}\left(\mathfrak{Q}_{m}\right), L_{p}(\mathfrak{Q}) / L_{p}\left(\mathfrak{Q}_{m}\right)\right) .
$$

An element $\phi^{(m)} \in \Lambda_{m}^{\prime}$ is a bounded linear transformation $\phi^{(m)}: L_{p}\left(\mathfrak{Q}_{m}\right) \rightarrow$ $L_{p}(\mathfrak{Q}) / L_{p}\left(\mathbb{Q}_{m}\right)$ which commutes with multiplication by $\mathbb{Q}_{m}$-simple functions, that is, $\phi^{(m)} A g=A \phi^{(m)} g$ whenever $A \in \mathbb{Q}_{m}$.

The Banach spaces $L_{p}^{(m)}(\mathfrak{Q})$ and $L_{p}(\mathfrak{Q}) / L_{p}\left(\mathfrak{Q}_{m}\right)$ are equivalent: each element $\tilde{\theta}$ of the quotient space $L_{p}(\mathfrak{Q}) / L_{p}\left(\mathscr{Q}_{m}\right)$ has a uniquely determined representative 
$\theta \in L_{p}(\mathfrak{Q})$ with $\mathscr{E}_{m} \theta=0$. Henceforth we shall identify an element of $L_{p}(\mathfrak{Q}) / L_{p}\left(\mathbb{Q}_{m}\right)$ with its canonical representative in $L_{p}^{(m)}(\mathfrak{Q})$; the norm, however, will be the quotient norm.

Now the event $A \in \mathbb{Q}_{m}$ may be regarded as an element of $L_{p}\left(\mathbb{Q}_{m}\right)$; so $\phi^{(m)} A$ may be viewed as a well-defined element of $L_{p}^{(m)}(\mathscr{Q})$. Abusing the notation, we may identify $\phi^{(m)}$ with a function which is locally in $L_{p}(\mathscr{Q})$ and satisfies $\mathcal{E}_{m} \phi^{(m)}=0$. The norm of the element $\phi^{(m)} A$ in $L_{p}(\mathfrak{Q}) / L_{p}\left(\mathbb{Q}_{m}\right)$ is

$$
\left\|\phi^{(m)} A\right\|=\inf _{\psi}\left\{\int_{A}\left|\phi^{(m)}-\psi\right|^{p} d \mu\right\}^{1 / p}
$$

where $\psi$ ranges over $L_{p}\left(\mathscr{Q}_{m}^{A}\right)$. If we write $\left\|\phi^{(m)}\right\|$ for the norm in $\Lambda_{m}^{\prime}$ we then have $\sup _{A}\left\|\phi^{(m)} A\right\| /\|A\|_{p} \leq\left\|\phi^{(m)}\right\|$ where $A$ ranges over $\mathcal{Q}_{m}$. The quantity on the left is

$$
N_{m}\left(\phi^{(m)}\right)=\sup _{A} \inf _{\psi}\left\{\int_{A}\left|\phi^{(m)}-\psi\right|^{p} d \mu / \mu(A)\right\}^{1 / p} .
$$

Thus we have $N_{m}\left(\phi^{(m)}\right) \leq\left\|\phi^{(m)}\right\|$. On the other hand it is easy to see that if $\phi^{(m)}$ is a function locally in $L_{p}(\mathfrak{Q})$ with $\tilde{E}_{m} \phi^{(m)}=0$ and $N_{m}\left(\phi^{(m)}\right)<\infty$ then $g \mapsto \phi^{(m)} g$, where $g \in L_{p}\left(\mathbb{Q}_{m}\right)$, defines a bounded linear transformation in $\Lambda_{m}^{\prime}$ with norm $\leq N_{m}\left(\phi^{(m)}\right)$. Thus. $\left\|\phi^{(m)}\right\|=N_{m}\left(\phi^{(m)}\right)$.

Let us form the Banach space product $\Lambda^{\prime}=\Pi_{m} \Lambda_{m}^{\prime}$. An element $\phi \in \Lambda^{\prime}$ has components $\phi^{(m)} \in \Lambda_{m}^{\prime}$ and $\|\phi\|=\sup _{m}\left\|\phi^{(m)}\right\|$. The subspace of $\Lambda^{\prime}$ consisting of those $\phi$ such that $\phi^{(n)}=\phi^{(m)}-\mathcal{E}_{n} \phi^{(m)}$ whenever $m \leq n$ is precisely the previously defined space $\mathrm{JN}_{p}$ and $\|\phi\|=\sup _{m}\left\|\phi^{(m)}\right\|=\sup _{m} N_{m}\left(\phi^{(m)}\right)={ }_{p}\|\phi\|_{\infty}$, since ${ }_{p}\|\phi\|_{\infty}$ was defined in $\S 2$ as $\sup _{m} N_{m}\left(\phi^{(m)}\right)$.

For a Banach space $H$, the space $\mathrm{JN}_{p}(H)$ of $H$-valued $\mathrm{JN}_{p}$ - "functions" is defined by the exact same procedure with $\Lambda_{m}^{\prime}$ replaced by

$$
\Lambda_{m}^{\prime}(H)=\operatorname{HOM}^{\mathfrak{Q}_{m}}\left(L_{p}\left(\mathfrak{Q}_{m} ; \mathbf{R}\right), L_{p}(\mathbb{Q}, H) / L_{p}\left(\mathbb{Q}_{m}, H\right)\right) .
$$

The proof of Proposition 2 in $\$ 5$ is now purely abstract. Suppose $X_{m}$ and $Y_{m}$ are Banach spaces which admit a ring $\mathfrak{Q}_{m}$ of endomorphisms. Then if we put $\Lambda_{m}=$

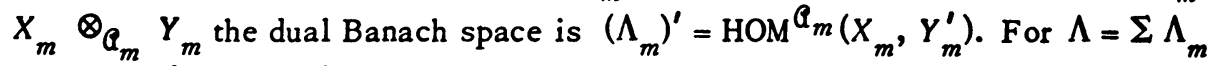
we get $(\Lambda)^{\prime}=\Pi\left(\Lambda_{m}\right)^{\prime}$, and finally, if $K$ is a (closed) subspace of $\Lambda$ then the dual of $\Lambda / K$ is the subspace of $(\Lambda)^{\prime}$ consisting of the elements which annihilate $K$. In the situation at hand we have ${ }_{p} L_{1}(H)=\Lambda / K$ where $X_{m}=L_{p^{\prime}}\left(\mathfrak{Q}_{m} ; \mathbf{R}\right)$ and $Y_{m}=$ $L_{p}^{(m)}(\mathscr{Q} ; H)$. If $p<\infty$ and $H^{\prime}$ is separable or reflexive then $Y_{m}^{\prime}=L_{p^{\prime}}\left(\mathscr{Q}_{;} ; H^{\prime}\right) / L_{p^{\prime}}\left(\mathbb{Q}_{m} ; H^{\prime}\right)$ and the subspace of $(\Lambda)^{\prime}$ which arises is exactly $\mathrm{JN}_{p^{\prime}}\left(H^{\prime}\right)$. In case $p=\infty$ or $H^{\prime}$ arbitrary, the space $L_{p^{\prime}}\left(\mathfrak{Q} ; H^{\prime}\right) / L_{p^{\prime}}\left(\mathfrak{Q}_{m} ; H^{\prime}\right)$ is only a subspace of $Y_{m}^{\prime}$; so $\Lambda_{m}^{\prime}\left(H^{\prime}\right)$ is merely a subspace of the dual of $\Lambda_{m}(H)$, and we can only assert that $\mathrm{JN}_{p^{\prime}}\left(H^{\prime}\right)$ is a subspace of the dual of ${ }_{p} L_{1}(H)$. 


\section{BIBLIOGRAPHY}

1. D. G. Austin, A sample function property of martingales, Ann. Math. Statist. 37 (1966), 1396-97. MR $33 \# 6687$.

2. D. L. Burkholder and R. F. Gundy, Extrapolation and interpolation of quasi-linear operators on martingales, Acta Math. 124 (1970), 249-304.

3. Burgess Davis, On the integrability of the martingale square function, Israel J. Math. 8 (1970), 187-190. MR 42 \#3863.

4. Charles Fefferman, Characterizations of bounded mean os cillation, Bull. Amer. Math. Soc. 77 (1971), 587-588. MR 43 \#6713.

5. C. Fefferman and E. M. Stein, $H^{p}$ spaces of several variables, Acta Math. 129 (1972), 137-193.

6. A. M. Garsia, The Burgess Davis inequalities via Fefferman's inequality, Ark. Mat. 11 (1973), 229-237.

7. R. K. Getoor and M. J. Sharpe, Conformal martingales, Invent. Math. 16 (1972), 271308.

8. C. S. Herz, Lipschitz spaces and Bernstein's theorem on absolutely convergent

Fourier transforms, J. Math. Mech. 18 (1968), 283-324.

9. F. John and L. Nirenberg, On functions of bounded mean os cillation, Comm. Pure Appl. Math. 14 (1961), 415-426. MR 24 \#A1348.

10. P. Lévy, Théorie de l'addition des variables aléatoires, 2ième édition, GauthierVillars, Paris, 1954.

11. P. -A. Meyer, Le dual de "H"" est "BMO" (cas continu), Séminaire de Probabilités VII, Lecture Notes in Math., vol. 321, Springer-Verlag, Berlin and New York, 1973, pp. 136-145.

DEPARTMENT OF MATHEMATICS, MCGILL UNIVERSITY, MONTREAL 101, QUEBEC, CANADA 